\title{
Treatment Failure After Image-Guided Percutaneous Radiofrequency Ablation (RFA) of Renal Tumors - A Systematic Review with Description of Type, Frequency, Risk Factors and Management \\ Therapieversagen nach bildgeführter perkutaner Radiofrequenzablation (RFA) von Nierentumoren - eine systematische Übersicht mit Beschreibung von Typ, Häufigkeit, Risikofaktoren und Management
}

\section{Authors}

Dominik Vollherbst ${ }^{1}$, Robert Bertheau², Hans-Ulrich Kauczor ${ }^{2}$, Boris Alexis Radeleff ${ }^{2}$, Philippe L. Pereira ${ }^{3}$, Christof-Matthias Sommer ${ }^{2}, 4$

Affiliations

1 Department of Neuroradiology, University Hospital Heidelberg, Heidelberg, Germany

2 Diagnostic and Interventional Radiology, University Hospital Heidelberg, Heidelberg, Germany

3 Clinic for Radiology, Minimally-invasive Therapies and Nuclear Medicine, SLK-Kliniken, Heilbronn, Germany

4 Clinic for Diagnostic and Interventional Radiology, Klinikum Stuttgart, Stuttgart, Germany

Key words radiofrequency ablation (RFA, RF ablation), tumor ablation (TA), image-guided, renal tumor, kidney

received 18.5.2016

accepted 6.8.2016

Bibliography

DOI http://dx.doi.org/10.1055/s-0042-115817

Published online: 14.09.2016 | Fortschr Röntgenstr 2017; 189: 219227 (c) Georg Thieme Verlag KG Stuttgart · New York

ISSN 1438-9029

Correspondence

Herr Dr. Christof-Matthias Sommer

Diagnostic and Interventional Radiology, University Hospital

Heidelberg

INF 110

69120 Heidelberg

Germany

Tel.: ++ 49/6221/5638534

Fax: $++49 / 6221 / 565720$

christof.sommer@med.uni-heidelberg.de

\section{ZUSAMMENFASSUNG}

Hintergrund Die Radiofrequenzablation (RFA) ist ein etabliertes Verfahren zur Behandlung von kleinen Nierentumoren. Das Ziel dieser Übersicht ist die systematische Erfassung von Häufigkeit, Typ, Risikofaktoren und Management von Therapieversagen nach bildgeführter perkutaner RFA von Nierentumoren.
Methode Zehn Studien (967 Patienten, 1033 Tumoren) mit einem mittleren/medianen Beobachtungszeitraum von $\geq 30$ Monaten wurden systematisch identifiziert und ausgewertet.

Ergebnisse und Schlussfolgerung Die bildgeführte perkutane RFA ist eine sehr effektive Technik zur Behandlung von umschriebenen Nierentumoren. Der residuelle nicht-abladierte Tumor ist der häufigste Typ des Therapieversagens (5,9\%), gefolgt von lokalem Tumorprogress $(4,7 \%)$. De-novo-Nierentumoren treten in 1,3\% und extrarenale Metastasen in 2,0 \% der Fälle auf. Lokaler Tumorprogress, de-novoNierentumoren und extrarenale Metastasen treten vorwiegend später als 12 Monate nach initialer RFA auf. Eine Tumorgröße $>3 \mathrm{~cm}$ und eine zentrale Tumorlokalisation sind die Hauptrisikofaktoren für das Therapieversagen. Im Falle eines Therapieversagens zeigt die erneute RFA eine hohe Erfolgsrate (86,3\% für residuelle nicht-abladierte Tumoren und $87,5 \%$ für lokalen Tumorprogress).

\section{Kernaussagen:}

- Therapieversagen kann in residuelle nicht-abladierte Tumoren und lokalen Tumorprogress unterteilt werden.

- Residuelle nicht-abladierte Tumoren treten in 5,9\% der Fälle auf.

- Lokaler Tumorprogress tritt in 4,7\% der Fälle auf.

- Tumorgröße und Tumorlokalisation sind Hauptrisikofaktoren für das Therapieversagen.

- Die erneute RFA ist effektiv und wird regelmäßig durchgeführt.

\section{ABSTRACT}

Background Radiofrequency ablation (RFA) is an established treatment for small renal tumors. The objective of this review is to systematically assess the type, frequency, risk factors and management of treatment failure after image-guided percutaneous RFA of renal tumors.

Method 10 studies (967 patients, 1033 tumors) with a mean/median follow-up of $\geq 30$ months were systematically identified and analyzed. Results and Conclusion Image-guided percutaneous RFA of localized renal tumors is very effective. The most common type of treatment failure is residual unablated tumor (5.9\%), followed by local tumor progression (4.7\%). De novo tumors in the kidneys occur in $1.3 \%$ of cases and extra-renal metastases in $2.0 \%$. Local tumor progression, de novo tumors in the kidneys and extra-renal metastases occur predominantly later than 12 months after initial RFA. Tumor size $>3 \mathrm{~cm}$ and central tumor location are the major risk factors for treatment failure. In the case of treatment failure, repeated RFA shows high success rates $(86.3 \%$ for residual unablated tumors and $87.5 \%$ for local tumor progression). 
Key Points:

- Treatment failure can be subdivided into residual unablated tumor and local tumor progression.

- Residual unablated tumor occurs in $5.9 \%$ of cases.

- Local tumor progression occurs in $4.7 \%$ of cases.

- Tumor size and location are the major risk factors for treatment failure.

- Repeated RFA is effective and commonly used for management.

\section{Citation Format}

- Vollherbst D, Bertheau R, Kauczor H et al. Treatment Failure After Image-Guided Percutaneous Radiofrequency Ablation (RFA) of Renal Tumors - A Systematic Review with Description of Type, Frequency, Risk Factors and Management. Fortschr Röntgenstr 2017; 189: 219-227

\section{Introduction}

Radiofrequency ablation (RFA) is an established treatment option for small renal tumors [1]. Due to the increasing number of diagnostic procedures, nowadays most renal tumors are detected incidentally at an early stage [1]. The currently available intermediate- and long-term data demonstrate a high rate of oncologic success of image-guided percutaneous RFA with 5-year cancer-specific survival rates of up to $100 \%[2,3]$. In the latest controlled trials, percutaneous RFA and nephrectomy show comparable survival rates. Takaki et al. compared percutaneous RFA with partial and radical nephrectomy, and reported 5-year cancer-specific survival rates of $100 \%$ [4]. Turna et al. also reported not significantly different cancer-specific survival rates between percutaneous RFA and partial nephrectomy (83.9\% vs. $100.0 \%$, respectively) but more cardiovascular complications in patients with long-term follow-up after nephrectomy [5]. The occasionally published superior overall survival in favor of patients undergoing nephrectomy may be explained by selection biases between the study groups (e. g. patient age and comorbidity) [6 - 8]. An alarming sign, however, is high local treatment failure rates of up to $35 \%$ in single-center RFA studies [9]. Beside residual unablated tumor and local tumor progression, recurrence outside the ablation zone in the kidneys and also extra-renal metastases have to be noted. Multiple single-center studies and meta-analyses investigated the outcome after image-guided percutaneous RFA of renal tumors but specific standardized data on mid- and longterm outcome is rare. Especially the data referring to treatment failure after percutaneous RFA of renal tumors is quite heterogeneous. This heterogeneity can be explained particularly by variable follow-up, different techniques and technologies as well as inhomogeneous reporting and non-standardized terminology. The objective of this review is to systematically assess the type, frequency, risk factors and management of treatment failure after image-guided percutaneous RFA of renal tumors in studies with a mean/median follow-up of $\geq 30$ months.

\section{Materials and Methods}

\section{Selection criteria and search strategy}

The systematic literature search and the selection and analysis of articles were conducted in accordance with the PRISMA statement [10]. Studies reporting on the oncologic outcome of image-guided percutaneous RFA of renal tumors with a mean or median follow-up of $\geq 30$ months were eligible for inclusion. Studies with insufficient data, a follow-up period of less than 30 months or studies with patient collectives published in more recent studies were excluded. Only studies written in English were analyzed. The search algorithm for MEDLINE (PubMed) was constructed using the following medical subject heading terms (MeSH) and text words: "radiofrequency ablation”, "RF ablation”, "RFA”, "kidney cancer”, "kidney tumor”, "renal cancer”, "renal mass”, “renal neoplasm”, "renal tumor”, "renal cell carcinoma”, "RCC”, “outcome" and "recurrence". The search was not restricted to years of publication or types of studies. After the systematic literature search, titles and abstracts were screened (primary selection) in order to find potentially appropriate articles. The next step was to perform a detailed evaluation of the main body of these studies (secondary selection) including cross-checking of the reference lists of the retrieved articles. The methodological quality of the studies potentially appropriate for inclusion was checked using the Downs and Black checklist for systematic reviews of non-randomized studies [11]. The search was completed during October 2015.

\section{Definitions}

According to Ahmed et al., residual disease in the ablation zone detected in the first follow-up imaging should be defined as "residual unablated tumor", and viable tumor in the ablation zone detected after an unremarkable first follow-up imaging should be defined as "local tumor progression" [12]. Accordingly, treatment failure was subdivided into (1) residual unablated tumor and (2) local tumor progression. Additionally, patients with de novo tumors in the kidneys and patients with extra-renal metastases were described.

\section{Results}

\section{Search results and baseline characteristics of the included studies}

After primary and secondary selection, 432 studies were identified. 10 studies published between 2010 and 2015 fulfilled the inclusion criteria and were analyzed in this systematic review. In total, data of 967 patients and 1033 treated tumors could be evaluated. The literature search flow and reasons for exclusion are presented in Fig. 1. An overview of the included studies is presented in $\nabla$ Table $\mathbf{1}$, and a summary of the results is presented in $>$ Table 2.

\section{Residual unablated tumor}

Across all included studies, 61 out of 1033 treated renal tumors showed residual unablated tumor after initial RFA, resulting in a 


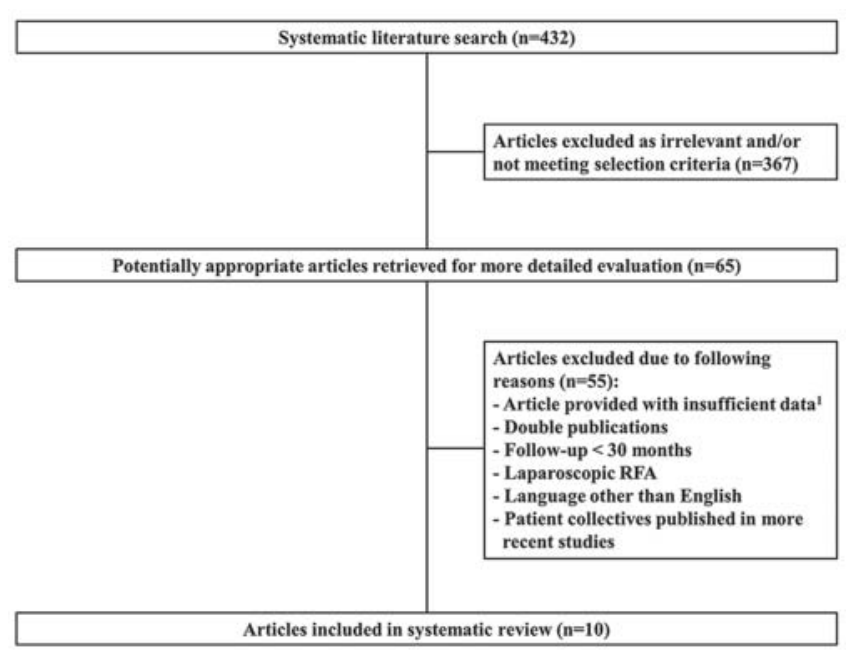

- Fig. 1 Literature search flow. Note: ${ }^{1}$ rate of residual unablated tumor or local tumor progression not indicated, incomplete description of the follow-up periods, type of image guidance not specified.

- Abb. 1 Flussdiagramm zur Erläuterung der Literaturrecherche. Legende: ${ }^{1}$ Häufigkeit von residuellen, nicht-abladierten Tumoren oder lokaler Tumorprogress nicht beschrieben, unvollständige Angabe der Beobachtungszeiträume, keine Spezifizierung der Methode zur Bildführung.

mean frequency of $5.9 \%$. The following risk factors were described: (1) tumor size [2, 3, 13 - 15], (2) central tumor location $[3,13-15],(3)$ a maximum treatment temperature of $\leq 70^{\circ} \mathrm{C}$ [15], and (4) clear cell subtype of renal cell carcinoma [15]. The cutoff for tumor size defined as a risk factor was between 3 and $4 \mathrm{~cm}[3,13-15]$. The results for the management of residual unablated tumor with repeated RFA are presented in $>$ Table 3 . In $54.1 \%$ of the cases $(n=33)$, residual unablated tumor was managed with repeated RFA. In $59.1 \%$, one additional session and in $40.9 \%$ two additional treatment sessions were performed. The treatment success rate, defined as no residual unablated tumor and no local tumor progression in the follow-up imaging, was $86.3 \%$.

\section{Local tumor progression}

The results for local tumor progression after image-guided percutaneous RFA are presented in $>$ Table 4 . Across all included studies, 48 out of 1033 treated renal tumors showed local tumor progression, resulting in a mean frequency of $4.7 \%$. In all but one case, imaging was the rationale for diagnosing local tumor progression. In this single case, viable tumor was found in a pretransplant nephrectomy specimen [16]. Local tumor progression occurred most frequently > 24 months after initial RFA (62.5\%; $n=10$ ). The mean time interval between initial RFA and diagnosis of local tumor progression was 28.0 months (range: 4 to 89 months). Risk factors described for local tumor progression were the same as for residual unablated tumor, with tumor size and tumor location as the most relevant risk factors [2, 3, 13 -15]. Local tumor progression was most frequently managed with repeated RFA $(47.8 \% ; n=48)$, followed by active surveillance $(12.5 \%$; $\mathrm{n}=6)$ and surgery $(10.4 \% ; \mathrm{n}=5)$. For the remaining patients (29.2\%; $n=14)$, management of local tumor progression was not described. The results for the management of local tumor progression with repeated RFA are presented in $>$ Table 5 . In the included studies, the outcome of the repeated RFA was given for 8 out of the 48 patients. The treatment success rate, defined as no residual unablated tumor and no local tumor progression in the following follow-up imaging, was $87.5 \%$.

\section{De novo tumors in the kidneys}

Across all included studies, 13 out of 967 treated patients showed de novo tumors in the kidneys, resulting in a mean frequency of $1.3 \%$. In all patients the de novo tumors were detected via radiological imaging. De novo tumors in the kidneys occurred most frequently between 13 and 24 months $(42.9 \% ; n=3)$ or later than 24 months (42.9\%; $n=3)$ after initial RFA. The mean time interval between initial RFA and diagnosis was 27.9 months (range: 11 to 48 months). Risk factors for de novo tumors in the kidneys were not specified in the included studies. The management of three de novo tumors was described $[13,14,17]$. Two of them were treated with repeated image-guided percutaneous RFA with a treatment success rate, defined as no residual unablated tumor and no local tumor progression in the following follow-up imaging, of $100 \%$.

\section{Extra-renal metastases}

Across all included studies, 19 out of 967 treated patients showed extra-renal metastases, resulting in a mean frequency of $2.0 \%$. Most extra-renal metastases occurred later than 24 months after initial RFA $(40.0 \%, n=4)$. The mean time interval between initial RFA and the diagnosis of extra-renal metastases was 22.5 months (range: 4 to 54 months). The most frequent locations of metastases were the lung, liver, bone, abdominal lymph nodes and pancreas. In the cases in which the outcome was reported, the mortality was high. Management or risk factors for patients with extra-renal metastases were not described in the included studies.

\section{Discussion}

According to our review, image-guided percutaneous RFA of small renal tumors is very effective. The results of our systematic review do not confirm the alarmingly high treatment failure rates published in very isolated single-center RFA studies. The frequencies of residual unablated tumor, local tumor progression, de novo tumors in the kidneys and extra-renal metastases are $5.9 \%$, $4.7 \%, 1.3 \%$ and $2.0 \%$, respectively.

Tumor size is a major risk factor for residual unablated tumor and for local tumor progression [2, 3, 13-15]. As presented above, treatment failure is more likely for tumors with a diameter of $>3.0 \mathrm{~cm}$ compared with smaller ones. The relevance of tumor size regarding the probability of tumor recurrence was published and discussed by Balageas et al. For tumors $\leq 4 \mathrm{~cm}$ (T1a), the recurrence-free survival rate was significantly higher than for tumors $>4 \mathrm{~cm}$ (T1b) (100\% vs. $57.1 \%$; $\mathrm{p}=0.0001)$ [14]. With the aid of pre-interventional embolization as an add-on to percutaneous RFA, the technical success, oncologic outcome and safety of RFA of bigger (T1b and T2), central and hardly visible renal 
- Table 1 Overview of the included studies: Study, patient and tumor characteristics.

- Tab. 1 Übersicht über die eingeschlossenen Studien: Studien-, Patienten- und Tumoreigenschaften.

\begin{tabular}{|c|c|c|c|c|c|c|c|c|}
\hline authors & year & $\begin{array}{l}\text { controlled (C) or } \\
\text { non-controlled } \\
\text { (NC) study design }\end{array}$ & $\begin{array}{l}\text { patients }(\mathrm{n}) / \\
\text { tumors }(\mathrm{n})\end{array}$ & $\begin{array}{l}\text { image } \\
\text { guidance }\end{array}$ & $\begin{array}{l}\text { mean or } \\
\text { median }{ }^{1} \\
\text { follow-up } \\
\text { (months) }\end{array}$ & $\begin{array}{l}\text { mean tumor } \\
\text { size } \\
(\mathrm{cm})\end{array}$ & $\begin{array}{l}\text { tumor location ( } n \text {, (relative } \\
\left.\text { frequency }{ }^{2}\right) \text { ): central/peri- } \\
\text { pheral/mixed }\end{array}$ & tumor histology (relative frequency) ${ }^{3}$ \\
\hline Ferakis et al. [13] & 2010 & NC & $31 / 39$ & CT & 61.2 & $3.1(1.3-7.5)$ & $35(89.7 \%) / 4(10.3 \%) / 0(0 \%)$ & - \\
\hline Sung et al. [20] & 2012 & $C 4^{4}$ & $40 / 45$ & CT & 36.6 & $2.4(1.0-6.0)$ & $36(90.0 \%) / 4(10.0 \%) / 0(0 \%)$ & $100 \%$ clear cell RCC \\
\hline Psutka et al. [2] & 2013 & NC & $185 / 185$ & CT & $77.2^{1}$ & $3.0(1.0-6.5)$ & $\begin{array}{l}139(75.1 \%) / 12(6.5 \%) / 34 \\
(18.4 \%)\end{array}$ & $\begin{array}{l}54.1 \% \text { clear cell, } 17.8 \% \text { chromophobe, } 2.7 \% \\
\text { oncocytic RCC }\end{array}$ \\
\hline Allen et al. [44] & 2013 & NC & $38 / 40$ & CT & 33.6 & $2.3(1.0-4.2)$ & $30(75.0 \%) / 10(25.0 \%) / 0(0 \%)$ & $61.1 \%$ RCC, $38.9 \%$ inconclusive or non-diagnostic \\
\hline Balageas et al. [14] & 2013 & NC & $62 / 71$ & $\mathrm{CT}, \mathrm{US}^{5}$ & 38.8 & $2.4(0.8-4.6)$ & $\begin{array}{l}57(80.3 \%) / 11(15.5 \%) / 3 \\
(4.2 \%)\end{array}$ & $\begin{array}{l}48.4 \% \text { clear cell, } 19.4 \% \text { papillary, } 11.3 \% \\
\text { cystic RCC }\end{array}$ \\
\hline Wah et al. [3] & 2014 & NC & $165 / 200$ & $\mathrm{CT}, \mathrm{US}^{6}$ & 46.1 & $2.9(1.0-5.6)$ & $\begin{array}{l}84(42.0 \%) / 16(8.0 \%) / 100 \\
(50.0 \%)\end{array}$ & $\begin{array}{l}80.0 \% \text { clear cell, } 7.0 \% \text { eosinophilic or } \\
\text { chromophobe, } 4.0 \% \text { papillary RCC }\end{array}$ \\
\hline Forauer et al. [16] & 2014 & NC & $39 / 46$ & CT & 35.5 & $2.6(1.2-4.0)$ & $46(100 \%) / 0(0 \%) / 0(0 \%)$ & $\begin{array}{l}59.0 \% \text { clear cell, } 35 \% \text { papillary, } 2 \% \text { mixed } \\
\text { type RCC }\end{array}$ \\
\hline Thompson et al. [21] & 2015 & $\mathrm{C}^{4}$ & $166 / 166$ & CT & $34.8^{1}$ & $2.1(-)$ & - & $\begin{array}{l}52.0 \% \text { clear cell, } 27.0 \% \text { papillary, } 4 \% \\
\text { chromophobe RCC }\end{array}$ \\
\hline Pieper et al. [17] & 2015 & NC & $38 / 38$ & CT & 54.6 & $2.1(-)$ & - & $\begin{array}{l}60.5 \% \text { clear cell RCC, } 13.2 \% \text { oncocytoma, } 10.5 \% \\
\text { angiomyolipoma }\end{array}$ \\
\hline lannuccilli et al. [15] & 2015 & NC & $203 / 203$ & CT & 34.1 & $2.5(1.0-6.0)$ & $\begin{array}{l}135(66.5 \%) / 12(5.9 \%) / 56 \\
(27.6 \%)\end{array}$ & $\begin{array}{l}47.3 \% \text { clear cell, } 20.2 \% \text { papillary RCC, } 10.3 \% \\
\text { oncocytoma }\end{array}$ \\
\hline Pooled data & $\begin{array}{l}2010- \\
2015\end{array}$ & s. a. & $967 / 1033$ & s. a. & $41.0^{1}$ & 2.5 & $\begin{array}{l}562(68.2 \%) / 69(8.4 \%) / 193 \\
(23.4 \%)\end{array}$ & $\begin{array}{l}56.7 \% \text { clear cell, } 11.8 \% \text { papillary, } 5.1 \% \\
\text { chromophobe RCC }\end{array}$ \\
\hline
\end{tabular}

"-“". no data available in the respective study; s. a. = see above (data not pooled): CT: computed tomography, US: ultrasound: RCC: renal cell carcinoma.

"-" Keine Angabe in der jeweiligen Studie; s. a. = siehe oben (Daten nicht statistisch zusammengefasst); CT: Computertomografie; US: Ultraschall; RCC: Nierenzellkarziom.

1 pooled data could only be calculated from the studies in which the mean was available.

gepoolte Daten konnten nur aus den Studien bestimmt werden, in denen der Mittelwert angegeben wurde.

2 on the basis of treated tumors.

auf Basis der behandelten Tumoren.

3 the three most frequent types of tumor histology are indicated, on the basis of tumors with histological examination.

die drei häufigsten histologischen Tumorarten sind angegeben, auf Basis der Tumoren mit histologischer Sicherung.

4 control group: partial nephrectomy.

Kontrollgruppe: partielle Nephrektomie.

${ }^{5}$ CT guidance: 60 patients, US guidance: 2 patients.

Bildführung mittels CT: 60 Patienten, Bildführung mittels US: 2 Patienten.

${ }^{6}$ CT guidance: 179 RFA procedures, US guidance: 31 RFA procedures.

Bildführung mittels CT: 179 Interventionen, Bildführung mittels US: 31 Interventionen. 
- Table 2 Overview of the results of the included studies.

- Tab. 2 Übersicht über die Ergebnisse der eingeschlossenen Studien.

\begin{tabular}{|c|c|c|c|c|}
\hline authors & $\begin{array}{l}\text { residual unablated tumor } \\
\left.\left.\text { (n (relative frequency }{ }^{1}\right)\right)\end{array}$ & $\begin{array}{l}\text { local tumor progression } \\
\left.\text { (n (relative frequency }{ }^{1}\right) \text { ) }\end{array}$ & $\begin{array}{l}\text { de novo tumors in the kidney } \\
\left.\text { (n (relative frequency }{ }^{2}\right) \text { ) }\end{array}$ & $\begin{array}{l}\text { extra-renal metastases } \\
\left.\text { (n (relative frequency }{ }^{2}\right) \text { ) }\end{array}$ \\
\hline Ferakis at al. [13] & $4(10.3 \%)$ & $3(7.7 \%)$ & $1(3.2 \%)$ & $0(0 \%)$ \\
\hline Sung et al. [20] & $8(17.8 \%)$ & $1(2.2 \%)$ & $0(0 \%)$ & $1(2.5 \%)$ \\
\hline Psutka et al. [2] & $24(13.0 \%)$ & $12(6.5 \%)$ & $5(2.7 \%)$ & $4(2.2 \%)$ \\
\hline Allen et al. [44] & $0(0 \%)$ & $0(0 \%)$ & $1(2.6 \%)$ & $0(0 \%)$ \\
\hline Balageas et al. [14] & $4(4.2 \%)$ & $2(2.8 \%)$ & $4(6.5 \%)$ & $4(6.5 \%)$ \\
\hline Wah et al. [3] & 9 (4.5\%) & $5(2.5 \%)$ & $0(0 \%)$ & $4(2.4 \%)$ \\
\hline Forauer et al. [16] & $0(0 \%)$ & $1(2.2 \%)$ & $0(0 \%)$ & $0(0 \%)$ \\
\hline Thompson et al. [21] & $0(0 \%)$ & $5(3.0 \%)$ & $0(0 \%)$ & $4(2.4 \%)$ \\
\hline Pieper et al. [17] & $2(5.3 \%)$ & $4(10.5 \%)$ & $2(5.3 \%)$ & $2(5.3 \%)$ \\
\hline lannuccilli et al. [15] & $11(5.4 \%)$ & $15(7.5 \%)$ & $0(0 \%)$ & $0(0 \%)$ \\
\hline pooled data & $61(5.9 \%)$ & $48(4.7 \%)$ & $13(1.3 \%)$ & $19(2.0 \%)$ \\
\hline \multicolumn{5}{|c|}{$\begin{array}{l}{ }^{1} \text { on the basis of treated tumors. } \\
\text { auf Basis der behandelten Tumoren. } \\
2 \text { on the basis of treated patients. } \\
\text { auf Basis der behandelten Patienten. }\end{array}$} \\
\hline
\end{tabular}

- Table 3 Management of residual unablated tumor with repeated RFA.

- Tab.3 Management von residuellen nicht-abladierten Tumoren mit erneuter RFA.

\begin{tabular}{|c|c|c|c|c|c|c|}
\hline \multirow[t]{2}{*}{ authors } & \multirow{2}{*}{$\begin{array}{l}\text { residual } \\
\text { unablated } \\
\text { tumor } \\
\text { (n) }\end{array}$} & \multirow{2}{*}{$\begin{array}{l}\text { tumors re-treated } \\
\text { with RFA } \\
\text { (n (relative } \\
\text { frequency)) }\end{array}$} & \multicolumn{2}{|c|}{$\begin{array}{l}\text { number of sessions for repeated } \\
\text { RFA }\end{array}$} & \multirow{2}{*}{$\begin{array}{l}\text { outcome of repeated } \\
\text { RFA -available data } \\
\left.\text { (n (relative frequency }{ }^{1}\right) \text { ) }\end{array}$} & \multirow{2}{*}{$\begin{array}{l}\text { outcome of repeated } \\
\text { RFA - treatment success }{ }^{2} \\
\text { (n (relative frequency)) }\end{array}$} \\
\hline & & & one session & two sessions & & \\
\hline Ferakis at al. [13] & 4 & $4(100 \%)$ & 3 & 1 & $4(100 \%)$ & $3(75.0 \%)$ \\
\hline Sung et al. [20] & 8 & $8(100 \%)$ & 3 & 5 & $8(100 \%)$ & $8(100 \%)$ \\
\hline Psutka et al. [2] & 24 & - & - & - & - & - \\
\hline Allen et al. [44] & 0 & - & - & - & - & - \\
\hline Balageas et al. [14] & 4 & $2(50.0 \%)$ & 2 & 0 & $2(100 \%)$ & $2(100 \%)$ \\
\hline Wah et al. [3] & 9 & $8(88.9 \%)$ & 5 & 3 & $8(100 \%)$ & $6(75.0 \%)$ \\
\hline Forauer et al. [16] & 0 & - & - & - & - & - \\
\hline Thompson et al. [21] & 0 & - & - & - & - & - \\
\hline Pieper et al. [17] & 2 & - & - & - & - & - \\
\hline lannuccilli et al. [15] & 11 & $11(100 \%)$ & - & - & $0(0 \%)$ & - \\
\hline pooled data & 61 & 33 (54.1\%) & $13 / 22^{3}(59.1 \%)$ & $9 / 22^{3}(40.9 \%)$ & 22 (66.7\%) & 19 (86.3\%) \\
\hline \multicolumn{7}{|c|}{$\begin{array}{l}\text { “-“: no data available in the respective study or no data indicated since no case of residual unablated tumor was reported in the respective study. } \\
\text { “-": Keine Angabe in der jeweiligen Studie oder keine Daten angegeben, da kein residueller nicht-abladierter Tumor in der jeweiligen Studie berichtet wurde. } \\
1 \text { on the basis of tumors re-treated with RFA. } \\
\text { definiert als kein residueller nicht-abladierter Tumor und keine lokale Tumorprogression in den Verlaufkontrollen nach erneuter Intervention. } \\
2 \text { defined as no residual unablated tumor and no local tumor progression in the follow-up imaging. } \\
3 \text { for } 11 \text { tumors the number of sessions was not indicated. } \\
\text { für } 11 \text { Tumoren wurde die Anzahl der Behandlungssitzungen nicht angegeben. }\end{array}$} \\
\hline
\end{tabular}


- Table 4 Local tumor progression.

- Tab.4 Lokale Tumorprogression.

\begin{tabular}{|c|c|c|c|c|c|c|c|c|c|}
\hline \multirow[t]{2}{*}{ authors } & \multirow{2}{*}{$\begin{array}{l}\text { local tumor } \\
\text { progression } \\
(n(\text { relative } \\
\left.\text { frequency }^{1}\right) \text { ) }\end{array}$} & \multirow{2}{*}{$\begin{array}{l}\text { rationale } \\
\text { for clas- } \\
\text { sifying } \\
\text { as local } \\
\text { tumor pro- } \\
\text { gression }\end{array}$} & \multicolumn{5}{|c|}{$\begin{array}{l}\text { time of diagnosis after initial RFA } \\
\text { (months; n (relative frequency)) }\end{array}$} & \multirow[t]{2}{*}{ risk factors } & \multirow{2}{*}{$\begin{array}{l}\text { management } \\
\text { (n (relative } \\
\text { frequency)) }\end{array}$} \\
\hline & & & $<6$ & $6-12$ & $13-24$ & $>24$ & $\begin{array}{l}\text { mean/median } \\
\text { (months) }^{2}\end{array}$ & & \\
\hline $\begin{array}{l}\text { Ferakis } \\
\text { et al. [13] }\end{array}$ & $\begin{array}{l}3 \\
(7.7 \%)\end{array}$ & imaging & $\begin{array}{l}0 \\
(0 \%)\end{array}$ & $\begin{array}{l}1 \\
(33.3 \%)\end{array}$ & $\begin{array}{l}1 \\
(33.3 \%)\end{array}$ & $\begin{array}{l}1 \\
(33.3 \%)\end{array}$ & 28.0 & $\begin{array}{l}\text { tumor size } \geq 4.0 \mathrm{~cm} \text {, } \\
\text { central tumor } \\
\text { location }\end{array}$ & $\begin{array}{l}\operatorname{RFA}(2(66.7 \%)) \\
\text { surgery }(1(33.3 \%))\end{array}$ \\
\hline $\begin{array}{l}\text { Sung } \\
\text { et al. [20] }\end{array}$ & $\begin{array}{l}1 \\
(2.2 \%)\end{array}$ & imaging & - & - & - & - & - & - & - \\
\hline $\begin{array}{l}\text { Psutka } \\
\text { et al. [2] }\end{array}$ & $\begin{array}{l}12 \\
(6.5 \%)\end{array}$ & imaging & - & - & - & - & 30.0 & tumor size $\geq 4.0 \mathrm{~cm}$ & $\begin{array}{l}\text { RFA }(6(50 \%)) \text {, } \\
\text { active surveillance } \\
(6(50 \%))\end{array}$ \\
\hline $\begin{array}{l}\text { Allen } \\
\text { et al. [44] }\end{array}$ & $\begin{array}{l}0 \\
(0 \%)\end{array}$ & - & - & - & - & - & - & - & - \\
\hline $\begin{array}{l}\text { Balageas } \\
\text { et al. [14] }\end{array}$ & $\begin{array}{l}2 \\
(2.8 \%)\end{array}$ & imaging & 0 & $\begin{array}{l}1 \\
(50.0 \%)\end{array}$ & $\begin{array}{l}1 \\
(50.0 \%)\end{array}$ & 0 & 18.0 & $\begin{array}{l}\text { tumor size } \geq 4.0 \mathrm{~cm} \text {, } \\
\text { central tumor } \\
\text { location }\end{array}$ & - \\
\hline $\begin{array}{l}\text { Wah } \\
\text { et al. [3] }\end{array}$ & $\begin{array}{l}5 \\
(2.5 \%)\end{array}$ & imaging & 0 & 0 & 0 & $\begin{array}{l}5 \\
(100.0 \%)\end{array}$ & 58.3 & $\begin{array}{l}\text { tumor size } \geq 3.0 \mathrm{~cm} \text {, } \\
\text { central tumor } \\
\text { location }\end{array}$ & $\begin{array}{l}\text { no management } \\
\text { with RFA }(0(0 \%))\end{array}$ \\
\hline $\begin{array}{l}\text { Forauer } \\
\text { et al. [16] }\end{array}$ & $\begin{array}{l}1 \\
(2.2 \%)\end{array}$ & $\begin{array}{l}\text { surgical } \\
\text { specimen }\end{array}$ & $\begin{array}{l}1 \\
(100 \%)\end{array}$ & 0 & 0 & 0 & 4.2 & - & surgery $(1(100 \%))$ \\
\hline $\begin{array}{l}\text { Thompson } \\
\text { et al. [21] }\end{array}$ & $\begin{array}{l}5 \\
(3.0 \%)\end{array}$ & - & 0 & $\begin{array}{l}1 \\
(20.0 \%)\end{array}$ & 0 & $\begin{array}{l}4 \\
(80.0 \%)\end{array}$ & 50.0 & - & - \\
\hline $\begin{array}{l}\text { Pieper } \\
\text { et al. [17] }\end{array}$ & $\begin{array}{l}4 \\
(10.5 \%)\end{array}$ & - & - & - & - & - & 25.7 & - & $\begin{array}{l}\text { no management } \\
\text { with RFA }(0(0 \%)) \text {, } \\
\text { surgery }(3(75.0 \%))\end{array}$ \\
\hline $\begin{array}{l}\text { Iannuccilli } \\
\text { et al. [15] }\end{array}$ & $\begin{array}{l}15 \\
(7.5 \%)\end{array}$ & imaging & - & - & - & - & 23.3 & $\begin{array}{l}\text { tumor size } \geq 3.5 \mathrm{~cm} \text {, } \\
\text { clear cell subtype of } \\
\text { RCC, maximum } \\
\text { treatment tempera- } \\
\text { ture } \leq 70^{\circ} \mathrm{C} \text {, non- } \\
\text { exophytic tumors }\end{array}$ & RFA (15 (100\%)) \\
\hline Pooled data & $\begin{array}{l}48 \\
(4.7 \%)\end{array}$ & s.a. & $\begin{array}{l}1 \\
(6.3 \%)\end{array}$ & $\begin{array}{l}3 \\
(18.8 \%)\end{array}$ & $\begin{array}{l}2 \\
(12.5 \%)\end{array}$ & $\begin{array}{l}10 \\
(62.5 \%)\end{array}$ & 28.0 & s.a. & $\begin{array}{l}\text { RFA }(23(47.9 \%)) \text {, } \\
\text { active surveillance } \\
(6(12.5 \%)) \text {, sur- } \\
\text { gery }(5(10.4 \%)) \text {, } \\
\text { not indicated } \\
(29.2 \%)\end{array}$ \\
\hline
\end{tabular}

“-“: no data available in the respective study or no data indicated since no case of local tumor progression was reported in this study; s. a. = see above, data not pooled; RCC: renal cell carcinoma.

“-": Keine Angabe in der jeweiligen Studie oder keine Daten angegeben, da keine lokale Tumorprogression in der jeweiligen berichtet wurde; s. a. = siehe oben (Daten nicht statistisch zusammengefasst); RCC: Nierenzellkarzinom.

1 on the basis of treated tumors. auf Basis der behandelten Tumoren.

2 pooled data could only be calculated from the studies in which the mean was available. gepoolte Daten konnten nur aus den Studien bestimmt werden, in denen der Mittelwert angegeben wurde. 
- Table 5 Management of local tumor progression with repeated RFA.

- Tab.5 Management von lokaler Tumorprogression mit erneuter RFA.

\begin{tabular}{|c|c|c|c|c|c|}
\hline authors & $\begin{array}{l}\text { local tumor } \\
\text { progression (n) }\end{array}$ & $\begin{array}{l}\text { tumors re-treated } \\
\text { with RFA ( }(\text { (relative } \\
\text { frequency)) }\end{array}$ & $\begin{array}{l}\text { outcome of repeated } \\
\text { RFA -available data } \\
\left.\text { (n (relative frequency }{ }^{1}\right) \text { ) }\end{array}$ & $\begin{array}{l}\text { mean follow-up } \\
\text { period (months) }\end{array}$ & $\begin{array}{l}\text { outcome of repeated } \\
\text { RFA - treatment } \\
\text { success }^{2} \text { ( } n \text { (relative } \\
\text { frequency)) }\end{array}$ \\
\hline Ferakis at al. [13] & 3 & $2(66.6 \%)$ & $2(100 \%)$ & 62.0 & $2(100 \%)$ \\
\hline Sung et al. [20] & 1 & - & - & - & - \\
\hline Psutka et al. [2] & 12 & $6(50.0 \%)$ & $6(100 \%)$ & 45.1 & $5(83.3 \%)$ \\
\hline Allen et al. [44] & 0 & - & - & - & - \\
\hline Balageas et al. [14] & 2 & - & - & - & - \\
\hline Wah et al. [3] & 5 & - & - & - & - \\
\hline Forauer et al. [16] & 1 & - & - & - & - \\
\hline Thompson et al. [21] & 5 & - & - & - & - \\
\hline Pieper et al. [17] & 4 & - & - & - & - \\
\hline lannuccilli et al. [15] & 15 & $15(100 \%)$ & $0(0 \%)$ & - & - \\
\hline Pooled data & 48 & $23(48.0 \%)$ & $8(34.8 \%)$ & 49.3 & 7 (87.5\%) \\
\hline \multicolumn{6}{|c|}{$\begin{array}{l}\text { “-“: no data available in the respective study or no data indicated since no case of local tumor progression was reported in the respective study. } \\
\text { “-”: Keine Angabe in der jeweiligen Studie oder keine Daten angegeben, da keine lokale Tumorprogression in der jeweiligen Studie berichtet wurde. } \\
1 \text { on the basis of tumors re-treated with RFA. } \\
\text { auf Basis der mittels RFA erneut behandelten Tumoren. } \\
2 \text { defined as no residual unablated tumor and no local tumor progression during the follow-up period. } \\
\text { definiert als kein residueller nicht-abladierter Tumor und keine lokale Tumorprogression in den Verlaufkontrollen nach erneuter Intervention. }\end{array}$} \\
\hline
\end{tabular}

tumors can be optimized [18-20]. Another major risk factor for treatment failure is a central tumor location [3, 13-15]. In the study of Wah et al., $77.8 \%$ of the residual unablated tumors were centrally located, whereas all exophytic or parenchymal tumors, regardless of size and location, were completely ablated in one RFA session [3].

According to our analysis, de novo tumors in the kidneys after image-guided RFA are rare but can occur up to 48 months after initial RFA. Compared with radical (contralateral kidney) and partial nephrectomy, the frequency of de novo tumors after treatment is comparable [21-23]. As synchronous renal cell carcinoma occurs with a frequency of up to $4.7 \%$, it is likely that some of the analyzed patients of this review had an occult tumor at the time of initial RFA [24]. The relevance of synchronous and metachronous renal tumors should not be underestimated in terms of cardiovascular-specific morbidity and mortality. As previously reported, preservation of renal function is extremely important to avoid cardiovascular events and hospitalization as well as to improve overall survival [25-27]. The manner in which image-guided RFA can be used very effectively for both tumor control and preservation of renal function has been demonstrated in multiple trials with high-risk patients (e. g. patients with single functioning kidneys and hereditary renal cell carcinoma [e. g. von Hippel Lindau disease]) [28-30].

Irrespective of the type and time of diagnosis of treatment failure, repeated RFA is an effective option for tumor control with a treatment success rate of $86.3 \%$ for residual unablated tumor and $87.5 \%$ for local tumor progression. The data supports the concept of multi-step RFA that is performed successfully for the treatment of T1a and T1b renal tumors with a very low major complication rate, preservation of renal function, high tumor control and a high rate of patient satisfaction. [1 - 3, 22, 30].

According to our review, extra-renal metastases after imageguided RFA of renal tumors are very rare but slightly more frequent than de novo renal tumors, and occur up to 4.5 years after initial RFA [3]. In a controlled study, the metastasis-free survival after treatment of T1a renal tumors was significantly better for surgery when compared with RFA $(p=0.005)$. However, the age at treatment and Charlson score - but not the treatment itself (partial nephrectomy vs. RFA) - were the independent predictors for overall survival [23]. In general, the outcome of patients developing metastatic disease after successful RFA of the primary renal tumor is often fatal, as it is the case after nephrectomy and during active surveillance [2, 3, $31-33]$.

Regarding the imaging follow-up after RFA of renal tumors, no generally accepted protocol has been identified. Different centers propose CT or MR imaging 1, 3, 6 and 12 months after RFA, and then annually for the following years [34-36]. The results of our review may specify the protocol: contrast-enhanced MRI of the kidneys 1, 3 and 6 months after RFA due to the rates of residual unablated tumors and local tumor progression, and afterwards annual imaging of the abdomen (contrast-enhanced MRI) and lung (non-enhanced CT) for 5 years due to the rates of local tumor progression, de novo tumors in the kidneys and extra-renal me- 
tastases. Also since image-guided percutaneous RFA is very effective and safe as a multi-step approach, the role of active surveillance in patients with localized renal tumors seems to be increasingly irrelevant [33, $37-39]$.

Besides RFA, percutaneous cryoablation is also commonly used. RFA and cryoablation are regarded as essentially equivalent for the treatment of small renal tumors $[40,41]$. The currently available mid- and long-term data indicate that the oncological outcome of both techniques is comparable, and depends more on patient selection and operator experience than on the ablation technique itself [ $40-44]$. The advantages of RFA are shorter ablation time, higher availability and excellent cost-effectiveness, whereas the advantages of cryoablation are direct visualization of the ablation zone ("ice ball") and lower rate of injury to the collecting system for larger central tumors [40, 41, 45].

This study has limitations. First, patients and tumors from different studies and institutions were analyzed, reducing the homogeneity of the included patients. Second, the included studies were conducted at some point during the last 6 years, a time in which the technical advantages and clinical experience of the relatively modern technology RFA have continuously improved. Third, the analyzed studies included not only patients with biopsy-proven renal cell carcinomas.

In conclusion, image-guided percutaneous RFA of localized renal tumors is very effective. Treatment failure can be subdivided into residual unablated tumor (occurring in $5.9 \%$ of cases) and local tumor progression (occurring in $4.7 \%$ of cases). Patients developing de novo tumors in the kidneys or extra-renal metastases after RFA are rare. Major risk factors for treatment failure are tumor size and location. For the management of both residual unablated tumor and local tumor progression, repeated RFA is commonly used and very effective.

Erratum (10.10.2016): Vollherbst D, Bertheau R, Kauczor $U$ et al. Treatment Failure After ImageGuided Percutaneous Radiofrequency Ablation (RFA) of Renal Tumors - A Systematic Review with Description of Type, Frequency, Risk Factors and Management. Fortschr Röntgenstr 2016; DOI: 10.1055/s-0042-115817

The number of treated patients was corrected in the abstract and in the results section (incorrect number: 1033 patients, correct number; 967 patients). Moreover, the number of treated renal tumors was corrected in the abstract (incorrect number: 967 renal tumors, correct number: 1033 renal tumors).

\section{Conflict of Interest}

No conflict of interest has been declared by the author(s).

\section{References}

[1] Sommer CM, Stampfl U, Kauczor HU et al. Percutaneous radiofrequency ablation of renal tumors: update 2015. Urologe A 2015; 54: 219-230

[2] Psutka SP, Feldman AS, McDougal WS et al. Long-term oncologic outcomes after radiofrequency ablation for $\mathrm{T} 1$ renal cell carcinoma. Eur Urol 2013; 63: 486-492

[3] Wah TM, Irving HC, Gregory W et al. Radiofrequency ablation (RFA) of renal cell carcinoma (RCC): experience in 200 tumours. BJU Int 2014; 113: $416-428$

[4] Takaki H, Yamakado K, Soga N et al. Midterm results of radiofrequency ablation versus nephrectomy for T1a renal cell carcinoma. Jpn J Radiol 2010; $28: 460-468$

[5] Turna B, Kaouk JH, Frota R et al. Minimally invasive nephron sparing management for renal tumors in solitary kidneys. J Urol 2009; 182: $2150-2157$

[6] Zagoria RJ, Traver MA, Werle DM et al. Oncologic efficacy of CT-guided percutaneous radiofrequency ablation of renal cell carcinomas. Am J Roentgenol 2007; 189: 429-436

[7] Salas N, Ramanathan R, Dummett S et al. Results of radiofrequency kidney tumor ablation: renal function preservation and oncologic efficacy. World J Urol 2010; 28: 583-591

[8] Zagoria RJ, Pettus JA, Rogers M et al. Long-term outcomes after percutaneous radiofrequency ablation for renal cell carcinoma. Urology 2011; 77: $1393-1397$

[9] McDougal WS, Gervais DA, McGovern FJ et al. Long-term followup of patients with renal cell carcinoma treated with radio frequency ablation with curative intent. J Urol 2005; 174: 61-63

[10] Liberati A, Altman DG, Tetzlaff J et al. The PRISMA statement for reporting systematic reviews and meta-analyses of studies that evaluate healthcare interventions: explanation and elaboration. BMJ 2009; 339: b2700

[11] Downs SH, Black N. The feasibility of creating a checklist for the assessment of the methodological quality both of randomised and non-randomised studies of health care interventions. J Epidemiol Community Health 1998; 52: $377-384$

[12] Ahmed M, Technology Assessment Committee of the Society of Interventional R. Image-guided tumor ablation: standardization of terminology and reporting criteria-a 10-year update: supplement to the consensus document. J Vasc Interv Radiol 2014; 25: 1706- 1708

[13] Ferakis N, Bouropoulos C, Granitsas T et al. Long-term results after computed-tomography-guided percutaneous radiofrequency ablation for small renal tumors. J Endourol 2010; 24: 1909-1913

[14] Balageas P, Cornelis F, Le Bras Y et al. Ten-year experience of percutaneous image-guided radiofrequency ablation of malignant renal tumours in high-risk patients. Eur Radiol 2013; 23: 1925-1932

[15] lannuccilli JD, Dupuy DE, Beland MD et al. Effectiveness and safety of computed tomography-guided radiofrequency ablation of renal cancer: a 14-year single institution experience in 203 patients. Eur Radiol 2015. DOI: 10.1007/s00330-015-4006-7

[16] Forauer AR, Dewey BJ, Seigne JD. Cancer-free survival and local tumor control after impendence-based radiofrequency ablation of biopsyproven renal cell carcinomas with a minimum of 1-year follow-up. Urol Oncol 2014; 32: 869-876

[17] Pieper CC, Fischer S, Strunk H et al. Percutaneous CT-Guided Radiofrequency Ablation of Solitary Small Renal Masses: A Single Center Experience. Rofo 2015; 187: 577-583

[18] Duan XH, Li YS, Han XW et al. C-arm CT-guided renal arterial embolisation followed by radiofrequency ablation for treatment of patients with unresectable renal cell carcinoma. Clin Radiol 2016; 71: 79-85 
[19] Winokur RS, Pua BB, Madoff DC. Role of combined embolization and ablation in management of renal masses. Seminars in interventional radiology 2014; 31: $82-85$

[20] Arima K, Yamakado K, Kinbara $\mathrm{H}$ et al. Percutaneous radiofrequency ablation with transarterial embolization is useful for treatment of stage 1 renal cell carcinoma with surgical risk: results at 2-year mean follow up. International journal of urology: official journal of the Japanese Urological Association 2007; 14: 585-590; discussion 590

[21] Tsui KH, van Ophoven A, Shvarts $\mathrm{O}$ et al. Nephron-sparing surgery for renal cell carcinoma. Rev Urol 1999; 1: 216-225

[22] Sung HH, Park BK, Kim CK et al. Comparison of percutaneous radiofrequency ablation and open partial nephrectomy for the treatment of sizeand location-matched renal masses. Int J Hyperthermia 2012; 28 : 227 234

[23] Thompson RH, Atwell T, Schmit G et al. Comparison of partial nephrectomy and percutaneous ablation for $\mathrm{CT} 1$ renal masses. Eur Urol 2015; 67: $252-259$

[24] Grimaldi G, Reuter V, Russo P. Bilateral non-familial renal cell carcinoma. Ann Surg Oncol 1998; 5: 548-552

[25] Kyung YS, You D, Kwon T et al. The type of nephrectomy has little effect on overall survival or cardiac events in patients of 70 years and older with localized clinical t1 stage renal masses. Korean J Urol 2014; 55 : $446-452$

[26] Huang WC, Levey AS, Serio AM et al. Chronic kidney disease after nephrectomy in patients with renal cortical tumours: a retrospective cohort study. Lancet Oncol 2006; 7: 735-740

[27] Weight C], Larson BT, Fergany AF et al. Nephrectomy induced chronic renal insufficiency is associated with increased risk of cardiovascular death and death from any cause in patients with localized cT1b renal masses. J Urol 2010; 183: 1317-1323

[28] Park BK, Kim CK, Park SY et al. Percutaneous radiofrequency ablation of renal cell carcinomas in patients with von Hippel Lindau disease: indications, techniques, complications, and outcomes. Acta Radiol 2013; 54 : $418-427$

[29] Nao T, Shimamoto T, Karashima T et al. Clinical study on patients with renal-cell carcinoma accompanied with Von Hippel-Lindau disease treated with radiofrequency ablation. Hinyokika Kiyo 2014; 60: 415 420

[30] Krokidis M, Spiliopoulos S, Jarzabek M et al. Percutaneous radiofrequency ablation of small renal tumours in patients with a single functioning kidney: long-term results. Eur Radiol 2013; 23: 1933-1939

[31] Brookman-May S, May M, Shariat SF et al. Features associated with recurrence beyond 5 years after nephrectomy and nephron-sparing sur- gery for renal cell carcinoma: development and internal validation of a risk model (PRELANE score) to predict late recurrence based on a large multicenter database (CORONA/SATURN Project). Eur Urol 2013; 64: $472-477$

[32] Buti S, Bersanelli M, Sikokis A et al. Chemotherapy in metastatic renal cell carcinoma today? A systematic review. Anticancer Drugs 2013; 24 : $535-554$

[33] Borghesi M, Brunocilla E, Volpe A et al. Active surveillance for clinically localized renal tumors: An updated review of current indications and clinical outcomes. Int J Urol 2015; 22: 432-438

[34] Cornelis F, Balageas P, Le Bras Y et al. Radiologically-guided thermal ablation of renal tumours. Diagn Interv Imaging 2012; 93: 246-261

[35] Haddad RL, Patel MI, Vladica P et al. Percutaneous radiofrequency ablation of small renal tumors using CT-guidance: a review and its current role. Urol ] 2012; 9: 629-638

[36] Georgiades C, Rodriguez R. Renal tumor ablation. Tech Vasc Interv Radiol 2013; 16: $230-238$

[37] Castaneda CV, Danzig MR, Finkelstein JB et al. The natural history of renal functional decline in patients undergoing surveillance in the DISSRM registry. Urol Oncol 2015; 33: 166 e117-e120

[38] Parker PA, Alba F, Fellman B et al. Illness uncertainty and quality of life of patients with small renal tumors undergoing watchful waiting: a 2-year prospective study. Eur Urol 2013; 63: 1122 - 1127

[39] Hwang EC, Yu HS, Kwon DD. Small renal masses: surgery or surveillance. Korean J Urol 2013; 54: 283-288

[40] Shin B], Chick JF, Stavropoulos SW. Contemporary Status of Percutaneous Ablation for the Small Renal Mass. Curr Urol Rep 2016; 17: 23

[41] El Dib R, Touma NJ, Kapoor A. Cryoablation vs radiofrequency ablation for the treatment of renal cell carcinoma: a meta-analysis of case series studies. BJU Int 2012; 110: 510-516

[42] Atwell TD, Schmit GD, Boorjian SA et al. Percutaneous ablation of renal masses measuring $3.0 \mathrm{~cm}$ and smaller: comparative local control and complications after radiofrequency ablation and cryoablation. Am J Roentgenol 2013; 200: 461 - 466

[43] Pirasteh A, Snyder L, Boncher N et al. Cryoablation vs. radiofrequency ablation for small renal masses. Acad Radiol 2011; 18: $97-100$

[44] Regier M, Chun F. Thermal Ablation of Renal Tumors: Indications, Techniques and Results. Dtsch Arztebl Int 2015; 112: 412 -418

[45] Higgins LJ, Hong K. Renal Ablation Techniques: State of the Art. Am J Roentgenol 2015; 205: 735-741 